\title{
3D Reconstruction of Property Right Body Based on E00
}

\author{
Lingling Zhang ${ }^{1}$, Yunfei Shi ${ }^{1, *}$, Zhenfeng $\mathrm{Cai}^{2}$, Changjiang Shao ${ }^{2}$ and Liming Lin $^{2}$ \\ ${ }^{1}$ School of Resource and Environment, Linyi University, 276000 Linyi,China \\ ${ }^{2}$ Linyi Bureau or Land Resources, 276000 Linyi, China \\ ${ }^{*}$ Corresponding author
}

\begin{abstract}
The property right body is the registration object for 3d cadastre. Its fast an economic reconstruction is the key to realizing the precision management of $3 D$ property rights. Most property rights are rule bodies and they have corresponding construction data and height information. The method of "stretching" can be applied to rebuild the property right bodies. This paper provides the pre-processing procedures of the $2 \mathrm{D}$ floor plans, analyzes the format of the EOO file and proposes the 3D reconstruction method based on E00 file. Also, there are practical cases, the result of which indicates that the algorithm is effective and viable.
\end{abstract} e00

Keywords-floor plans; property right body; $3 D$ reconstruction;

\section{INTRODUCTION}

The management of space right is one of the heated research topics now. In recent years, Guo[1,2], Stoter $[3,4]$ and other scholars have introduced the $3 \mathrm{D}$ cadastre to solve the problems in space right management. That means the property right body (PRB) with clear 3D ownership boundaries will be regarded as the registration body to manage $3 \mathrm{D}$ rights. The PRB is the smallest unit in 3D cadastre. Zhan[5]think that $\mathrm{PRB}$ is equal to the parcel in $2 \mathrm{D}$ cadastre, which is often expressed in polyhedrons. Registration in the form of property right bodies can accurately record the space ownership boundaries of the property units, which is an effective way to confirm the space right. However, the total of PRBs in the city is very large. Therefore, its fast and economic reconstruction is the key to realize the precision management of 3D property rights. But most property right bodies in reality are rule bodies, with corresponding construction data (floor plans) and height information. The 3D reconstruction technology based on 2D graphic method(Tao,2005) can be applied to establish the 3D model of PRB, which means that we regard the 2D graphs as the undersurface and establish the body model by "stretching" from bottom to the top according to given height. In this paper, the finish construction survey and schematic map of the buildings were treated as original data, from which the 3D PRB data have been generated through affine transformation, data rising and other operations.

\section{PRE-PROCESSING OF 2D DATA}

The construction drawings are what the construction process is based on. It exists extensively in all construction design units and has the characteristics of high positional accuracy and clear definitions. In the drawing, the geometric, semantic and other information, both inside and outside the building, has been specifically described, so that the drawing is suitable for the $3 \mathrm{D}$ reconstruction of the building. In this paper, we reconstruct the $3 \mathrm{D}$ model according to the finish construction survey and schematic map of the buildings. During the process, the coordinates and elevation of the $3 \mathrm{D}$ data came from the coordinates and elevation of those corner points in the drawing for the bottom of the building (the first floor of the building). The schematic maps of the floors, on the other hand, provided the structure of all floors. Based on these schematic structural maps, the 3D data of the PRB of each floor could be generated. There weren't any coordinates in the schematic map of the floors (the elevation can be acquired from the finish construction survey). Besides, the length-to-width ratio may not be the same, so pre-processing was needed. Here, the criterion for the pre-processing was the drawing for the basement. By translation, zooming and other affine transformation procedures, we revised the floor structural drawing. After adjusting, the control points on the floor structural drawing should have the same $\mathrm{x}$, y coordinates as those of the same name on the base drawing. The control points of the same name refer to the corresponding spatial locations in the real world. After projection in the direction of the ground, they should match. Usually, we take the corner points of the buildings, the corner points at the staircases, or other points with characteristics as control points of the same name. In this experiment, the ArcInfo package has been applied to adjust the structural drawings of each floor. The Figure 1 shows the adjusting procedures.

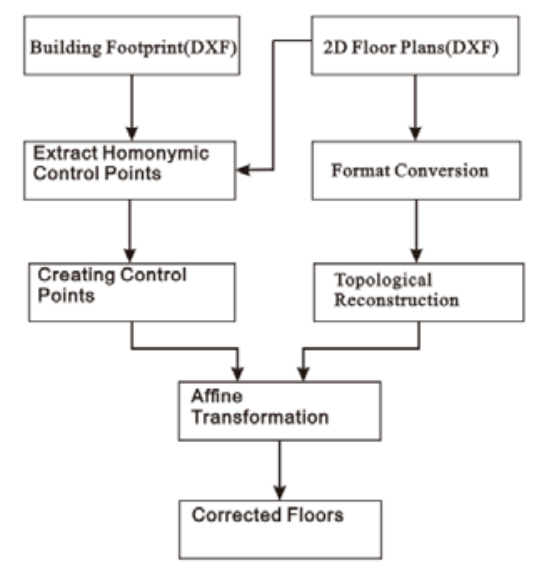

FIGURE I. FLOW-PROCESS DIAGRAM OF FLOOR STRUCTURAL DRAWING ADJUSTMENT 
(1) Choosing the control points of the same name is the key for the adjusting process. In order to achieve conformity between the floor plans and the base drawings of each floor after adjusting, in each building, at least four control points of the same name should be chosen. Starting from the base drawing, vertically stretch the four chosen points and intersect them with the floor plans. The intersection points are corresponding control points of the same name of this floor from the base drawing. Therefore, when choosing these control points, the structural drawings of every floor should be taken into consideration. The points that did not exist in the structural drawings of some floors should not be chosen. For example, in Figure 2, (a) is the base drawing of the building. 11 of the corner points have been chosen as potential control points of the same name and 4 of them will finally be chosen. In revised drawing (b), we have much more choices. We can choose any 4 of the 11 points as the control points of the same name for adjusting. But in order to achieve conformity with drawing (c), we can only choose from point 1-8. Similarly, in order to achieve conformity with drawing (d), we can only choose the four points: point 5, 6, 7 and 8. Therefore, at last, we determined that point $5,6,7$ and 8 were the control points of the same name.

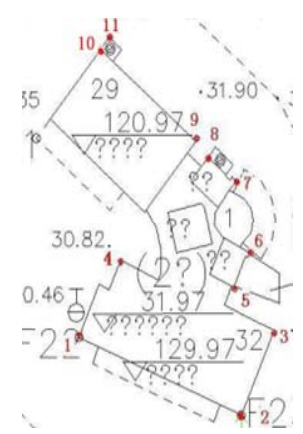

(a)

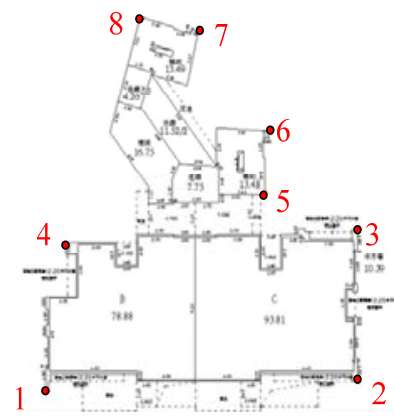

(c)

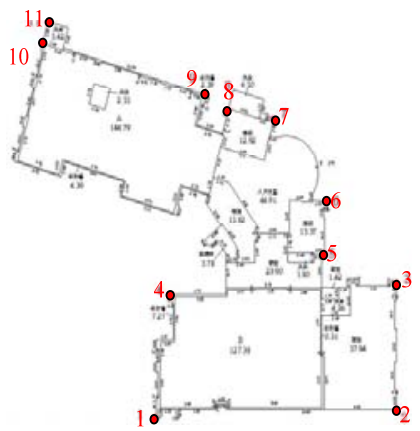

(b)

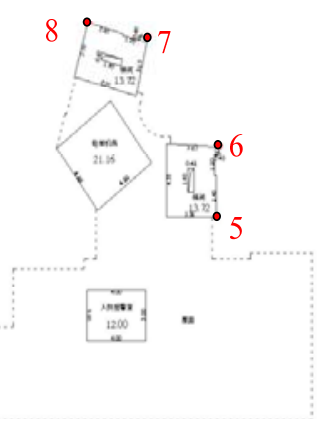

(d)
FIGURE II. CHOOSING THE CONTROL POINTS OF THE SAME NAME

(2) Format change. Apply the order of ArcInfo to change the dxf floor structural drawings into Coverage format.

(3) After the format change, there will be four control points in the Coverage format floor structural drawing (marked by red oval shape in Figure 3(a)). Because we use new control points of the same name to revise this floor plan, we need to eliminate these four control points and avoid the data problems caused by the format change, such as data loss, or increase, and so on. The red line in Figure 3 (a) is the internal boundary generated in the changing process. By eliminating the excess data and rebuilding the topological relations, the standardized floor structural drawings would be generated.
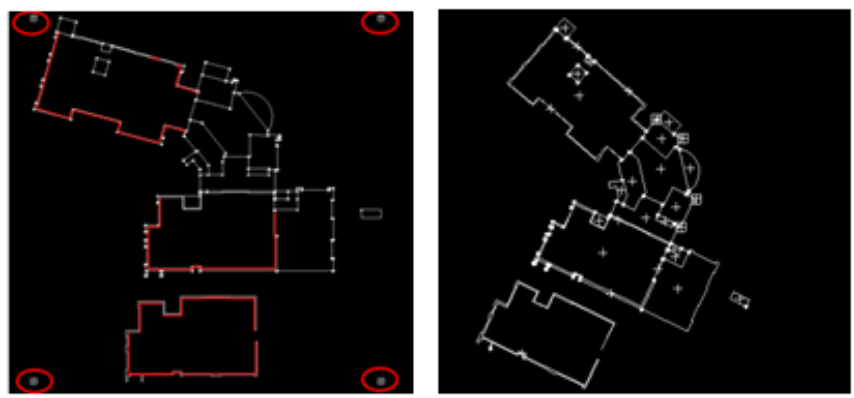

(a)Processing of the floor structural drawing (b)Floor plan after adjusting

FIGURE III. ADJUSTING OF FLOOR STRUCTURAL DRAWING

(4) Create the control point file. Add the coordinates of the four control points of the same name into the control point file.

(5) Affine transformation. Use the file for control points of the same name to implement affine transformation.

\section{3D RECONSTRUCTION BASED ON E00}

After the affine transformation, the floor plans of each floor got revised. After adjusting, the coordinates and relevant parameters in the floor structural drawing are the same as those in the base drawing. We can imagine the revised floor plans as the floor slab in the building. We give elevation value to all these floor slabs. As a result, these floor slabs will float right above the base at different heights, just like a building whose walls have been torn down but the building hasn't collapsed, with the floor slabs floating in the air. Therefore, the pre-processing of the data from the floor structural drawings actually only built the floor slabs of the building. The next step would be to establish the walls of each floor so that we can reconstruct the PRB of each floor.

One E00 file is made of different parts, and each part is referred as one section. For example: ARC section, CNT section, LAB section, PAL section and INFO section, etc[6]. One section is also referred as one file, which matches a physical file of Coverage format. Specific information is included in each section. For example, the ARC section includes the information of the segmental arc; the CNT section includes the information of the centroid of polygon; and the LAB section includes the notes. In our application, two parts should be focused: ARC section and PAL section. In these two sections, the ARC section stores the information of the segmental arc. It records the ID of the segmental arc, the ID of the starting and ending points, the ID of the polygon to the left of the arc followed by the ID of the polygon to the right, and the information of the vertexes, which decide the shape of the arc, in proper order. The vertex is not topological primitive and does not get involved in the topological construction, and there is no ID for the vertex, therefore only the coordinates $(\mathrm{x}, \mathrm{y})$ of each vertex have been given. The PAL section stores information of polygon. Polygon appears through quoting the 
segmental arcs in the ARC section. For a polygon, the minimum bounding rectangle MBR (referred as Envelope in ArcGIS) is first recorded. Then the information of the segmental arcs which form the polygon is recorded. Three types of information of each arc have been included: the ID of the segmental arc, the ID of the starting point of the arc and the ID of the polygon which shared the arcs with this polygon. There is one thing worth mentioning: the ID of the segmental arc can be negative.

Essentially, the segmental arc in E00 is polylines, which is formed by multiple interconnected line segments. When one polyline creates the 3D wall, each line segment will be "stretched" as a patch. Therefore, each segmental arc should be broken at each vertex. $\mathrm{N}$ topological sides will be generated from one arc. $\mathrm{N}=\mathrm{n}+1$ and $\mathrm{n}$ is the number of vertex in the segmental arc. If one segmental arc is decided by $\mathrm{n}$ vertexes, there will be $n+2$ points if including the starting and ending points and $n+1$ linear segments. Each segment will be one 3D topological side, therefore, there will be $n+1$ topological sides. During the process, we should also record the segmental arcs and their corresponding sides. Such information will be useful when these polygons generate patches.

Each polygon will generate a patch. During the process, the patch is made up of sides, while these sides are composed of broken segmental arcs of the polygons. Due to the fact that the segmental arcs forming the polygons in E00 files are in an anti-clockwise direction, we can just organize the sides based on the order of the segmental arcs. At this time, sides will naturally be organized in an anti-clockwise direction. Now we are going to explain the process using a very simple example. As shown in Figure 4: A and B are two nodes. In order to distinguish them from the vertexes, we mark them with black solid circles and capital letters. The rest of them are vertexes, which are used to decide the shape of the arcs and marked with small letters. There are three segmental arcs in this figure: $\mathrm{e} 1=\{\mathrm{A}, \mathrm{a}, \mathrm{b}, \mathrm{B}\}, \mathrm{e} 2=\{\mathrm{A}, \mathrm{c}, \mathrm{d}, \mathrm{B}\}$ and $\mathrm{e} 3=\{\mathrm{A}, \mathrm{f}, \mathrm{e}, \mathrm{B}\}$. The starting and ending point of each arc are nodes, while the middle part is the vertex. There are two polygons: $F 1=\{$ e1,- e3 $\}$ and $\mathrm{F} 2=\{-\mathrm{e} 2, \mathrm{e} 3\}$. The minus sign in front of the segmental arc means that the reverse direction will be applied. After breaking the segmental arcs in this figure, eight nodes (nodes and vertexes in Coverage will all be transformed into nodes) and nine sides (each line in Coverage will be generated as on side) will be generated. They are: E1 =(a,A); E2 =(a,b); E3 =(b,B); $\mathrm{E} 4=(\mathrm{A}, \mathrm{f}) ; \mathrm{E} 5=(\mathrm{f}, \mathrm{e}) ; \mathrm{E} 6=(\mathrm{e}, \mathrm{B}) ; \mathrm{E} 7=(\mathrm{B}, \mathrm{d}) ; \mathrm{E} 8=(\mathrm{A}, \mathrm{c}) ; \mathrm{E} 9$ $=(c, d)$. There will be two patches: one polygon generates one patch, they are: $\mathrm{F} 1=\{-\mathrm{E} 1, \mathrm{E} 2, \mathrm{E} 3,-\mathrm{E} 6,-\mathrm{E} 5,-\mathrm{E} 4\}$; $\mathrm{F} 2=\{\mathrm{E} 8, \mathrm{E} 9,-\mathrm{E} 7,-\mathrm{E} 6,-\mathrm{E} 5,-\mathrm{E} 4\}$.

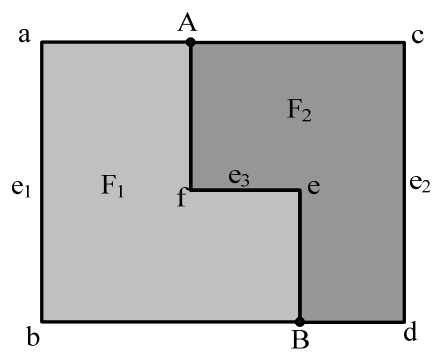

FIGURE IV. ORGANIZATION MODE OF COVERAGE
By applying the above-mentioned approach to 2D floor plans, the topological data of this floor can be generated. The next procedure is to generate the side face and body data by referring to the information in the structural drawing. After transforming E00 data, the dimension of 2D floor plans has been increase to $3 \mathrm{D}$ level. During the process, each vertex in the 2D floor plans will become one vertical side, which will generate one vertical patch. And each polygon will generate one body. The Figure 5 is an example of generating 3D PRB using this approach.

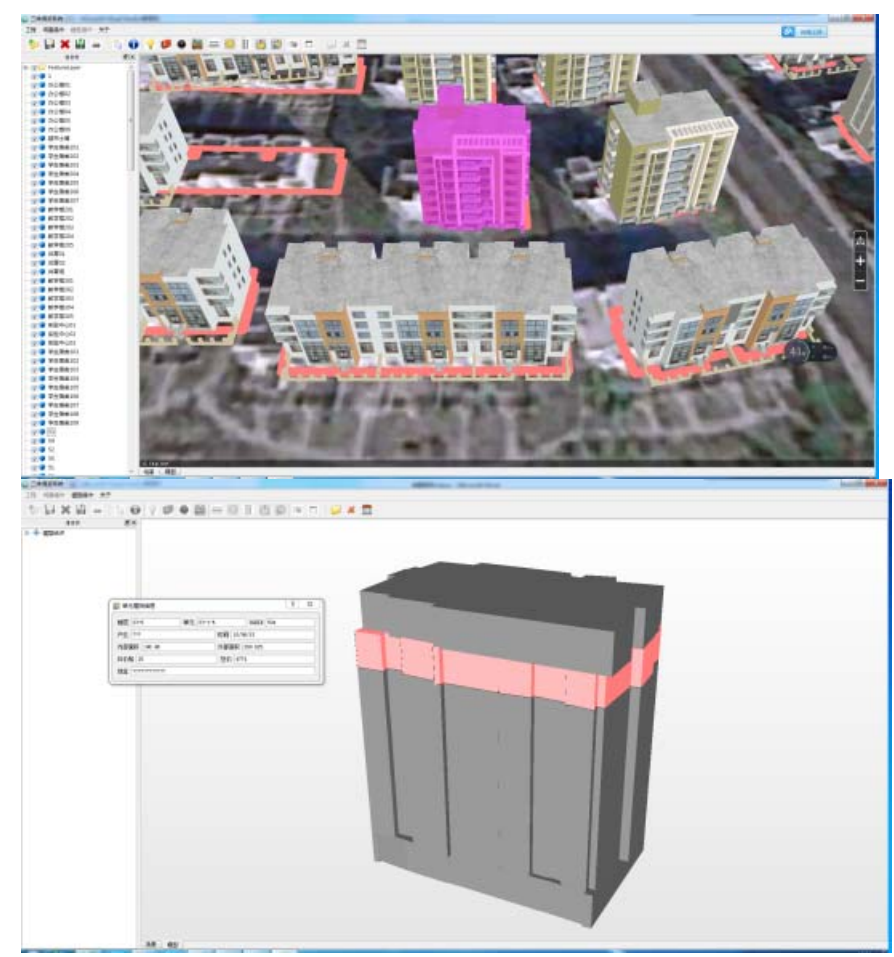

FIGURE V. 3D RECONSTRUCTION OF PRB BASED ON E00 DATE

\section{CONCLUSION}

It is an economic and effective way to rebuild the PRB through the "extrusion" of 2D graphs, thus enjoys great potential in future application. In this paper, we have had some positive try outs of this technology. However, before the reconstruction, much pre-processing of the data from the $2 \mathrm{D}$ floor plans is required. For example, we need to revise the points of the same name in various floors according to the outline of the base floor, filter the layer, and do scale-zooming, etc. These processing procedures are seemingly trivial but essentially significant. Often, problems of these procedures will lead to the failure of the algorithm in later period. And such processing costs the most labor, material resources and time. Therefore, it is necessary to study a pre-processing system, which can realize the automatic capture, adjusting, scale-changing, layer extraction, and other automated pre-processing procedures based on the architectural base drawing. If there were any breakthroughs in that technology, the working proficiency will be greatly improved and the cost of 3D property right management will be largely reduced. This is the direction of future studies. 


\section{ACKNOWLEDGEMENTS}

This work was financially supported by the National Natural Science Foundation of China (Grant No. 41201407).

\section{REFERENCES}

[1] Guo R.Z, Li L., Ying S., Luo P., He B., Jiang R.R.. Developing a 3D cadastre for the administration of urban land use: A case study of Shenzhen, China, Computers, Environment and Urban Systems. 40(2013)46-55.

[2] Guo R.Z, Ying S. Form Analysis and Data Expression of 3D Cadastre. China Land Science, 24(2010)45-51.

[3] STOTER J. E., Van Oosterom P. Technological Aspects of a 3D Cadastral Registration. International Journal of Geographical Information Science,19(2005)669-696.

[4] Stoter J E, Ploeger H. D..Property in 3D-Registration of Multiple Use of Space: Current Practice in Holland and the Need or a 3D Cadastre. Computers, Environment and Urban Systems , 27 (2003)553-570.

[5] Zhan C.G, Qi Z.G., Zhao J.H. Analysis on Establishing the 3D Cadastre. Scientific and Technological Management of Territorial Resouces,23(2006)79-81.

[6] Information on http://www.digitalpreservation.gov/formats/fdd/ fdd000291.shtml \#identification 